\title{
EFFECT OF nm-THIN INORGANIC LAYERED FILLERS ON the Crystallization of Polymer Nanocomposites
}

\author{
H. Nakajima, Z.M. Wang, K. Strawhecker, and E. Manias ${ }^{1}$ \\ Materials Science and Engineering Department, Pennsylvania State University, \\ 325-D Steidle Bldg, University Park, PA 16801
}

\begin{abstract}
The crystallization behavior of polymers in nanocomposites with inorganic fillers (montmorillonite layered silicate, MMT) is reviewed. Various different polymers are comparatively discussed [poly(vinyl alcohol) (PVA), polypropylene (PP), syndiotactic-polystyrene (sPS), and poly(ethylene oxide) (PEO)] representing three types of filler/matrix interactions: strong specific interactions (PVA/MMT), weak/negligible interactions (sPS and PP/o-MMT), and "unfavorable" (PEO/MMT). In the case of PVA/MMT, crystallization of PVA is strongly promoted by MMT, also stabilizing a new crystal form not found in bulk PVA. For sPS and PP/o-MMT, crystallization is only moderately affected, exhibiting traces of simple heterogeneous nucleation and mostly bulk-like crystal structures, with very small traces on non-bulk crystals. For PEO, crystallization is impeded near the MMT surfaces, due to coordination of the surface cations to the PEO. In all cases smaller spherulite sizes develop when filler is added, independent of the size of the bulk polymer spherulites, whereas the crystallization temperature changes reflect the strength of the polymer/surface interactions.
\end{abstract}

\section{INTRODUCTION}

The very large commercial importance of polymers has been driving an intense investigation of polymeric composites reinforced by particulates, fibers, and layered inorganic fillers. In particular, in the case of layered inorganic fillers, talc and mica have traditionally attracted the most interest. However, recent advances in polymer/clay and polymer/layered-silicate nanocomposite materials ${ }^{1,2}$ have inspired efforts to disperse clay-based fillers in almost any polymer available, usually expecting that complete exfoliation of the inorganic fillers in the polymer would yield the best performing systems ${ }^{2}$.

At the same time, the crystallization of polymers next to these inorganic surfaces has not been studied as extensively, and not beyond a few systems (e.g. nylon-6 and montmorillonite ${ }^{3}$ ). The nylon-6/montmorillonite studies have shown a nucleating and epitaxial effect of the inorganic surfaces, which stabilizing a surface-induced crystalline phase ${ }^{3}(\gamma$ crystal), that is rarely found in the bulk polymer. This effect has been used to enhance the mechanical and thermal properties of the polymer, since the surface nucleated crystalline phase has better mechanical and thermal characteristics than the bulk crystal phases. For the nylon- 6 case, the current opinion is that the origin of this substantial effect on the polymer

\footnotetext{
${ }^{1}$ address correspondence to: manias@psu.edu
} 
crystallization lies in the manner that amide interact with the inorganic aluminosilicates, through strong hydrogen-bonding interactions.

The aim of this work is to reveal the general effects of the dispersion of such nano-layered fillers on polymer crystallization, and also to unveil the origins of the polymer-specific effects by tracing them back to the molecular characteristics of the polymers. Along these lines, we compare the crystallization behavior of polymer/montmorillonite nanocomposites, across a number of different polymers; namely, poly(vinyl alcohol) $\left[\mathrm{PVA}^{4}\right]$, polypropylene $\left[\mathrm{PP}^{5}\right]$ and syndiotactic-polystyrene $\left[\mathrm{sPS}^{6}\right]$, and poly(ethylene oxide) $\left[\mathrm{PEO}^{7}\right]$.

\section{RESULTS AND DISCUSSION}

In a first approach, we focus on how the crystal nature and crystal morphology change in polymers in the immediate vicinity of nm-thin montmorillonite (MMT) surfaces. Four different polymers, with qualitatively and quantitatively varied interactions with MMT, have been investigated: Specifically, in descending order of interaction strength:

(a) poly(vinyl alcohol) $\left(\mathrm{PVA}^{4}\right)$, which interacts with very strong specific interactions (i.e., hydrogen bonding) with the silicon-oxide surface of montmorillonite;

(b) poly(ethylene oxide) (PEO ${ }^{7}$ ), which is bound on the MMT surfaces via a crown-ether coordination to the alkali cations $\left(\mathrm{Li}^{+}, \mathrm{Na}^{+}\right.$, etc) sorbed on the MMT surfaces;

(c) syndiotactic-polystyrene (sPS ${ }^{6}$ ), which interacts via polar (dipole-dipole) interactions with alkyl-imidazolium MMT; and

(d) polypropylene $\left(\mathrm{PP}^{5}\right)$, which has very weak interactions with alkyl-ammonium MMT, thus in this case revealing effects mostly due to geometric/topological constraints.

In figure 1, we show the crystal morphology for PP, PEO, and sPS. Our studies revealed a few common changes in the polymer crystallinity across these polymers. Namely, across all polymers studied, when $\mathrm{nm}$-thin inorganic MMT layers are dispersed in a polymer, its crystallites generally grow to much smaller sizes than in the respective bulk polymer (fig. 1). This decrease in crystallite size originates from the geometric constraints that the inorganic MMT fillers impose to the polymer, which result in nucleation of vastly larger number of crystallites typically of low spherical symmetry (non-spherulitic crystal morphologies), and occurs independently of the polymer-inorganic interactions. In the extreme case, when these geometric constraints become comparable to the monomer size (i.e., when polymer is intercalated -inserted- in between parallely-stacked layered fillers separated by $1-3 \mathrm{~nm}$ ) all polymer crystallization vanishes. The above behavior is general across all polymers studied here, as well as for other polymers reported in literature, and is expected to be the general behavior of semi-crystalline polymers next to nm-thin inorganic layered-fillers. 

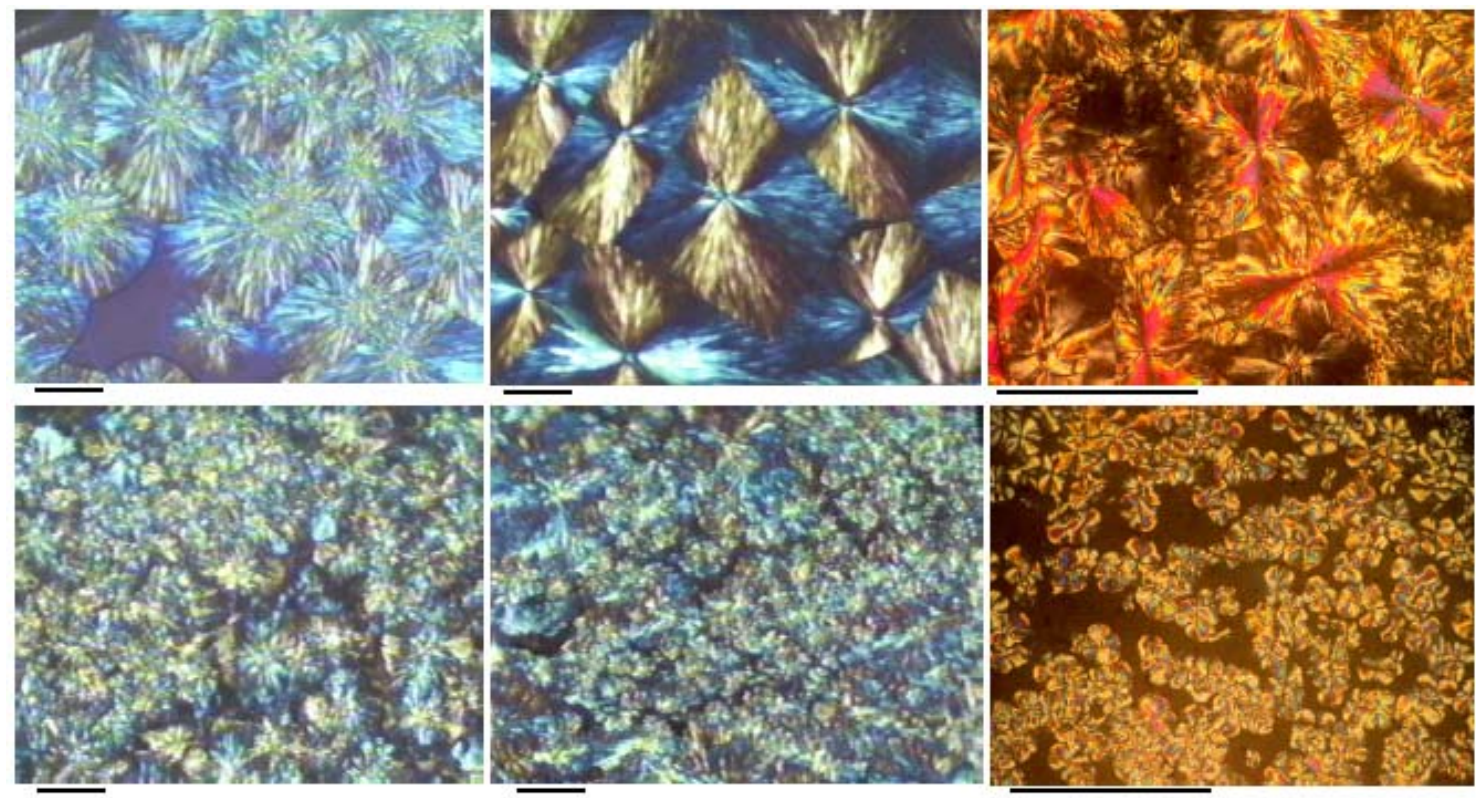

Figure 1. The general effect of layered nm-thin fillers on the polymers crystallization is exemplified by the comparison of the crystal morphologies of neat/unfilled polymers [top row] versus their respective nanocomposites [bottom row] containing 3wt\% layered-silicate filler. From left to right, three different polymers are compared: polypropylene [left], poly(ethylene oxide) [center], and syndiotactic-polystyrene [right].In all cases the scale-bar denotes $100 \mu m$. Adopted from [5,7,6].

Beyond this common behavior, a number of polymer-specific effects were observed, which originate from the particular manner that each polymer interacts with the inorganic surfaces. In the general case, where some attractive interactions exist (e.g., PP, sPS), the inorganic particles heterogeneously nucleate polymer crystals similar in nature to those seen in the respective neat polymers ${ }^{5,6}$. This is the most common behavior, and has been reported in literature for numerous polymers. In those special cases, where there exist strong/specific interactions between the polymer and the inorganic surface (e.g. nylon- $6^{3}$ and PVA $^{4}$ ) a new crystal structure is formed next to the MMT surfaces, at the expense of the bulk-like crystal $^{3,4}$. This new "epitaxially" grown phase has a different crystal unit cell (as revealed by XRD), and a higher melting temperature (as revealed by DSC), compared to the neat -unfilled- polymer ${ }^{3,4}$. Such drastic changes in the crystal nature are due to the very strong interactions between these particular polymers and MMT.

Independent of whether the heterogeneously nucleated crystal is the same or different in crystal symmetry than the bulk-polymer crystals, the general behavior of nucleation is demonstrated in figure 2, which follows in time the growth of a PVA crystal nucleated by a montmorillonite tactoid. For PEO the polymer/inorganic attractive interactions are mediated by the montmorillonite's cations to which PEO coordinates; in this case, although the $\mathrm{PEO} /$ alkali-cation coordination is promoting the dispersion of the inorganics in the polymer matrix, at the same time it amorphizes the polymer in the vicinity of MMT since 

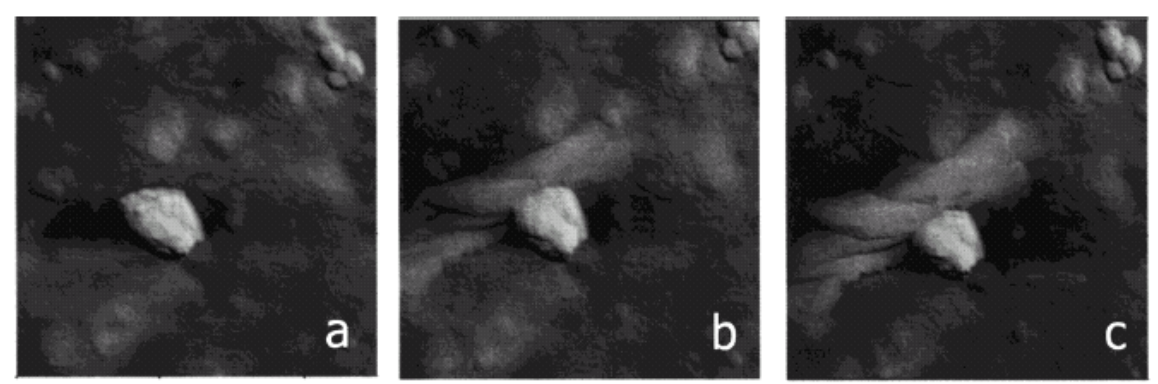

Figure 2. A time series of a PVA crystal in the vicinity of $a$ protuberant inorganic filler tactoid. The PVA C
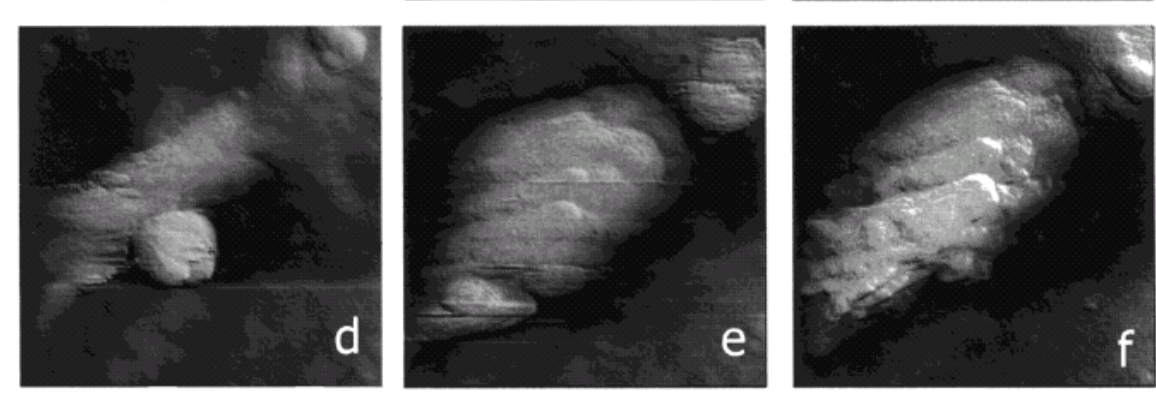

crystal initiates next to the inorganic surface (a), grows in size (b-d), and eventually covers completely the surface of the silicate tactoid (e). Fig from [4].

Figure 3. Time series of a PEO crystal growth. The box in (a) and (f) outlines the area shown in (b)-(e) at higher mag., and focus on the growth of a spherulite "front" as it encounters an MMT agglomerate. The scale bar in all images is $10 \mu \mathrm{m}$. Fig from [7].
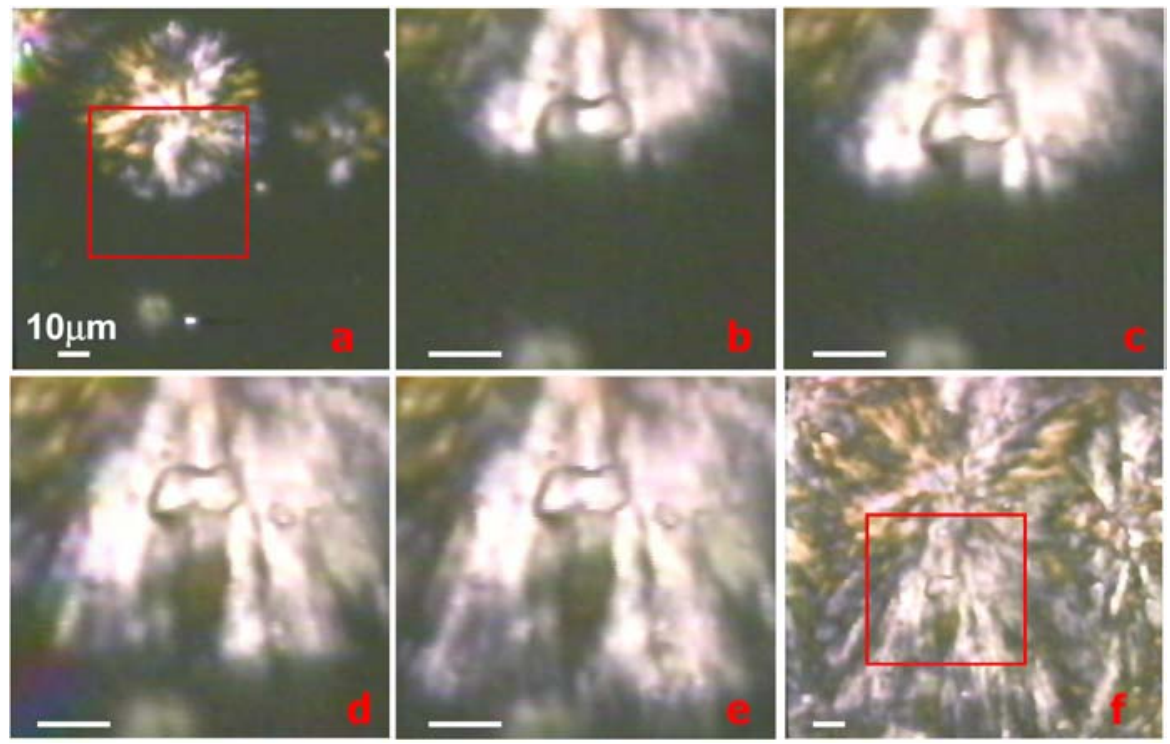

it promotes crown-ether conformations across the chain. Consequently, heterogeneous nucleation by the MMT layers is highly unprobable and the vast majority of PEO crystallites nucleate far away from the inorganic surfaces ${ }^{7}$; furthermore, crystal growth is hindered whenever the growth front encounters an alkali-cation-bearing MMT surface (figure 3). This last behavior is only observed for ethylene-oxide containing polymers, where the coordination interactions are strong enough to promote polymer/silicate miscibility but are not supportive of the polymer crystallization ${ }^{7}$.

This nucleating effect is also reflected in the scanning DSC studies, when the nanocomposites are compared with the respective bulk polymers (figure 4). In those cases where the polymer crystals are heterogeneously nucleated by the inorganics, the $\mathrm{T}_{\mathrm{c}}$ peak during the cooling cycle is shifted towards higher 

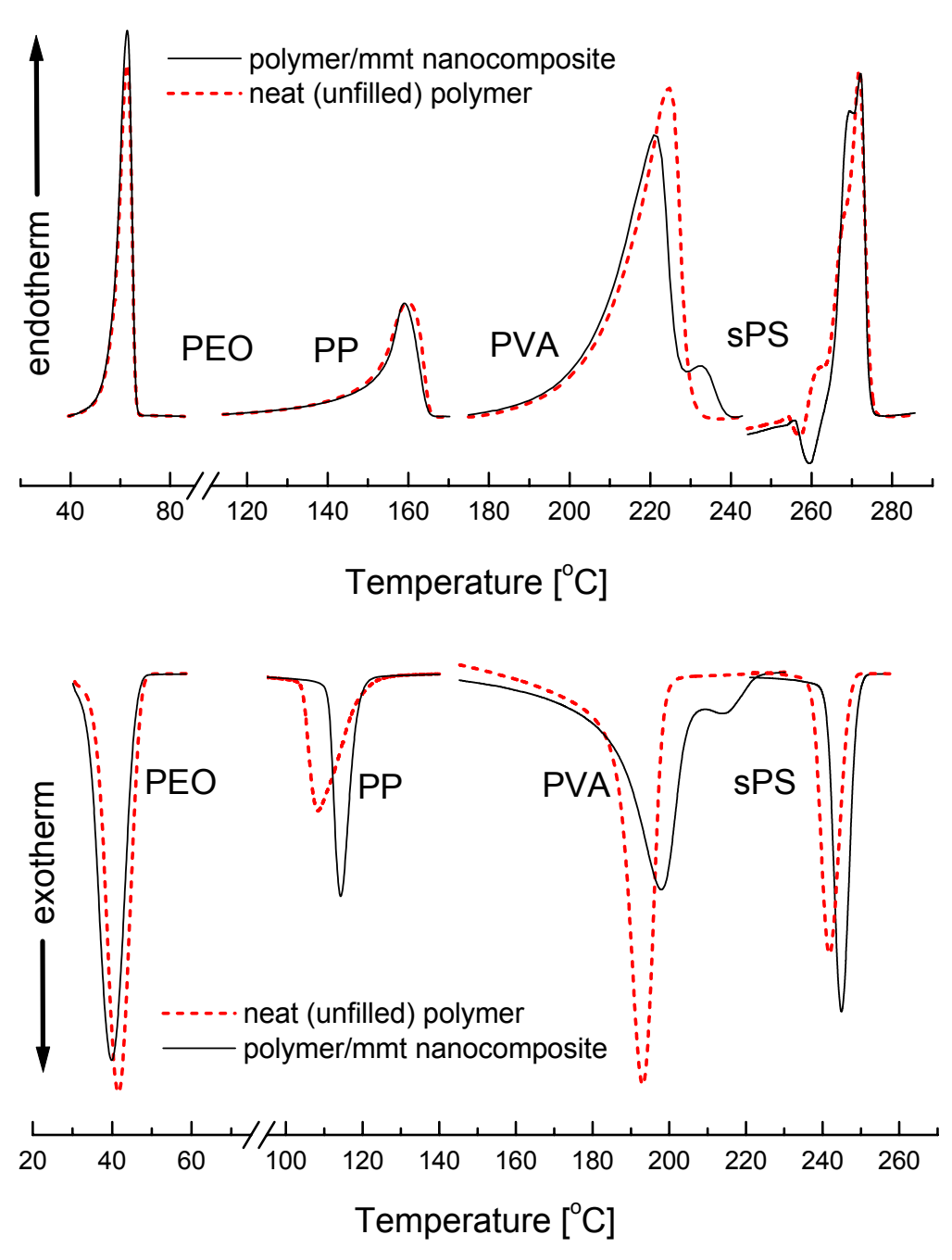

Figure 4. Scanning DSC of the neat/unfilled polymers and their nanocomposites, for four different polymers: poly(ethylene oxide) [PEO], polypropylene [PP], poly(vinyl alcohol) [PVA], and syndiotactic-polystyrene [sPS].

[top] DSC heating traces, $c f$. melting points; [bottom] DSC cooling traces, cf. crystallization temperatures. All scans done at $10^{\circ} \mathrm{C} / \mathrm{min}$.

For most polymers the Tm remains largely unaffected by the nanolayered fillers, except in those cases where there occur epitaxial crystals (e.g. PVA), or crystal polymorphisms (e.g. sPS). In contrast, the Tc is altered in most cases, reflecting the effect of the fillers on crystal nucleation.

temperatures and simultaneously becomes sharper than the corresponding bulk (e.g. fig 4, cf. PP, sPS); this behavior is also present independent of whether a new crystal form is promoted by the inorganic fillers (e.g. fig 4, cf. PVA). In contrast, for those polymers that montmorillonite hinders crystallization the $\mathrm{T}_{\mathrm{c}}$ peak shift to lower temperatures (e.g. fig 4, cf. PEO) compared to the respective bulk (unfilled) polymer.

During the same scanning DSC experiments (figure 4) the effect of the dispersion of such nano-layers in the polymers is also shown. Typically, there effect on the $T_{m}$ peak is small, if any at all, since the $\mathrm{T}_{\mathrm{m}}$ peak is characteristic of the crystal symmetry, lamella size, etc and largely insensitive on effects of nucleation and of crystallite morphology (e.g. fig 4, $c f$. PP, PEO). There only exists a measurable difference in the $T_{m}$ peak in those cases where the filler-induced crystals are of different crystal symmetry (e.g. PVA, where a higher $\mathrm{T}_{\mathrm{m}}$ appears ${ }^{4}$ ), or in those cases where the thermal history of the nanocomposite preparation gives rise to polymorphisms in the polymer crystal (e.g. sPS, where the high-temperature annealing during nanocomposite formation results in $\alpha / \beta$ polymorhpism ${ }^{6}$ ). 
In order to quantify the kinetics of crystallization, isothermal crystallization measurements were also carried out. The overall crystallization rate is the product of the nucleation rate and the crystal growth rate. Therefore, in order to evaluate the effect of clay addition on the crystal growth rate, the measurements were performed at undercoolings below but close to the polymer's $\mathrm{T}_{\mathrm{c}}$, where the nucleation rates are relatively invariant of temperature. Figure 5 shows the half times of crystallization $\left(\mathrm{t}_{1 / 2}\right)$ for various isothermal crystallization temperatures ( $\left.\mathrm{T}_{\text {iso }}\right)$ for PP-MA and PP-MA $/ 5 \mathrm{wt} \%$ organo-montmorillonite nanocomposites (the half time of crystallization $\left(\mathrm{t}_{1 / 2}\right)$ is defined as the necessary time to reach $50 \%$ of total crystallinity). As expected, $\mathrm{t}_{1 / 2}$ increased with as $\mathrm{T}_{\text {iso }}$ approached the $\mathrm{T}_{\mathrm{c}}$. However there were no distinct difference between PP-MA and its nanocomposite, in contrast with previous studies ${ }^{8}$. Given that, as mentioned above, the clay addition results in the increase of nucleation density and the number of nucleated spherulites per unit area increases by more than 10-fold, even at low inorganic concentrations (fig. 1), these results indicate that there should be a decrease of the linear growth rate due to the clay addition by $c a$. 10-fold. Similar isothermal crystallization studies for PEO and sPS nanocomposites are reported in detail elsewhere ${ }^{7,9}$, and largely reach the same conclusion.

Summarizing, even in the simplest approach the comparative study of polymer crystallization in the vicinity of nm-scale inorganic surfaces reveals a very rich behavior, that includes general effects common across all polymers, as well as polymer-specific responses. Elucidating the origins of these responses is necessary, if one is to develop generally applicable design paradigms for polymer/nanoscopic-inorganic systems and composites.

\section{REFERENCES}

[1] E. P Giannelis, R. Krishnamoorti, E. Manias, Advances in Polymer Science, 138:107 (1998)

[2] M. Alexandre, P. Dubois, Mater. Sci. Eng. R, 28:1 (2000)

[3] D.M. Licoln, R.A. Vaia, Z.G. Wang, B.S. Hsiao, R. Krishnamoorti, Polymer, $42: 9975$ (2001)

[4] K. Strawhecker, E. Manias, Macromolecules, 34:8475 (2001); Chem. Mater., 12:2943 (2000)

[5] E. Manias, A. Touny, L. Wu, K. Strawhecker, B. Lu, T.C. Chung, Chem. Mater., 13:3516 (2001)

[6] Z.M. Wang, T.C. Chung, J.W. Gilman, E. Manias J.Polym.Sci. B: Polym. Phys. 41:3173 (2003)

[7] K. Strawhecker, and E. Manias Chemistry of Materials 15:844 (2003).

[8] P. Maiti, P.H. Nam, M. Okamoto, N. Hasegawa, A. Usuki, Macromolecules, 35:2042 (2002)

[9] T.-M. Wu, S.-F.; Hsu, J.-Y. Wu, J Polym Sci Part B:Polym Phys 41:560 (2003) 